Proc. 13th International School on Theoretical Physics: Symmetry and Structural Properties of Condensed Matter \title{
Spin-Charge Conversion Effects and Anomalous Optical Responses of Spin-Orbit Systems
}

\author{
G. TATARA ${ }^{a, b, *}$ \\ ${ }^{a}$ RIKEN Center for Emergent Matter Science (CEMS), Wako, Saitama, Hirosawa, 2-1, 351-0198, Japan \\ ${ }^{b}$ RIKEN Cluster for Pioneering Research (CPR), Wako, Saitama 351-0198, Japan

\begin{abstract}
In this short review, spin-charge conversion effects induced by spin-orbit interaction are discussed from theoretical viewpoints. Spin Hall effect is formulated in terms of a single response function of spin density for an applied electric field, where the diffusion equation is not required for description and no ambiguity associated with spin current arises. The spin-charge conversion effects meaning the mixing of the electric and magnetic fields lead to various anomalous optical responses, such as negative refraction and directional dichroism, as shall be studied in detail for the Rashba spin-orbit interaction case.
\end{abstract}

DOI: 10.12693/APhysPolA.135.1215

PACS/topics: spintronics, spin-charge conversion, electromagnetic cross correlation, metamaterial, Doppler shift

\section{Spin-charge conversion}

Spin-charge conversion is the central concept of spintronics. The spin Hall effect is written as a response of current to the applied electric field $\boldsymbol{E}$ as

$$
j_{\mathrm{s}, j}^{k}=\theta_{\mathrm{sh}} \epsilon_{i j k} E_{i}
$$

with $\theta_{\text {sh }}$ being a constant. Here the flow of the spin current $j_{\mathrm{s}}$ is in the $j$-direction and spin polarization is along $k$-direction, $\theta_{\mathrm{sh}}$ is a constant, and $\epsilon_{i j k}$ is the totally-antisymmetric tensor. For the inverse spin Hall effect, formula connecting input and output currents, $j_{i}=\theta_{\text {ish }} \epsilon_{i j k} j_{\mathrm{s}, j}^{k}$, was postulated in Ref. [1], for conveniently explaining experimental results, where $\boldsymbol{j}$ denotes the observed electric current. The relation is of course a phenomenological one, and physically not correct. For instance, charge current is conserved while spin current is not, so they cannot be related simply. Nevertheless, the conversion formula is convenient to interpret experimental results and is widely used.

The spin accumulation generated by an applied electric field in the presence of the Rashba spin-orbit interaction [2], argued originally by Dyakonov and Perel [3], is called the Rashba-Edelstein effect [4]. Its inverse effect, the inverse Rashba-Edelstein effect, corresponds to a charge current generation as a result of spin accumulation, instead of spin current. The effect was experimentally observed for the case of interface Rashba spin-orbit interaction [5] and discussed theoretically in Ref. [6]. The apparent difference between the spin Hall and Rashba-Edelstein effects is that the former describes spin current, while the latter is discussed in terms of spin accumulation.

Theoretically, the standard spin Hall effect and Rashba-Edelstein effect arise from the spin-orbit interaction having different symmetries, more precisely, whether

\footnotetext{
*corresponding author; e-mail: gen.tatara@riken.jp
}

they are even or odd in the momentum operator. For spin-orbit interaction even in the wave vector, correlation functions between currents, no matter whether they are electric or spin, have finite spatially uniform component, while for odd interaction, correlation function between current and density has finite uniform component. It may appear reasonable, therefore, to regard spin Hall and Rashba-Edelstein effects as the effects generating spin current and spin density, respectively, by a driving electric field.

This distinction is, however, physically meaningless as spin density and spin currents are related to each other by a continuity equation and spin density is induced whenever spin current is induced. Moreover, spin current representation has a serious fundamental issue of being not an observable. In other words, its definition is theoretically not unique because it is a non-conserved current. Observation of generated spin current is therefore carried out only by detecting spin density like in Ref. [7]. Theoretically, spin Hall effect has been described by combining spin Hall conductance, which relates spin current and applied electric field, and a diffusion equation for spin, describing the spin accumulation formed as a result of the generated spin current. The diffusion equation is usually treated phenomenologically, and the issue of the ambiguity of spin current is therefore smeared out in those descriptions.

\subsection{Spin continuity equation}

The diffusion equation is equivalent to the continuity equation

$$
\dot{s}_{\alpha}+\nabla \cdot j_{\mathrm{s}}^{\alpha}=\mathcal{T}_{\alpha}
$$

where $s$ and $\mathcal{T}$ denote induced spin density and spin relaxation torque, respectively, and the divergence is with respect to the spatial direction of flow. The relaxation torque is expressed as proportional to the induced nonequilibrium spin density and spin relaxation time $\tau_{\mathrm{r}}$ as 


$$
\mathcal{T}=\frac{\boldsymbol{s}}{\tau_{\mathrm{r}}}
$$

This relation, usually phenomenologically assumed, has been justified in some cases [8, 9]. From Eqs. (2) and (3), a steady nonequilibrium spin configuration is determined by the balance of flow and relaxation of spin as

$$
s_{\alpha}=\tau_{\mathrm{r}} \nabla \cdot \boldsymbol{j}_{\mathrm{s}}^{\alpha} .
$$

Generated spin current is thus detectable by measuring nonequilibrium spin accumulation. One must note, however, that definition of spin current is not unique, as we can add any contribution $\delta \boldsymbol{j}_{\mathrm{s}}^{\alpha}$ to spin current and $\nabla \cdot \delta \boldsymbol{j}_{\mathrm{s}}^{\alpha}$ to the relaxation torque without modifying the continuity equation. In other words, the form of the spin current and relation torque depends on the experimental detection scheme, but this fact is disregarded in phenomenological arguments.

\subsection{Current-spin correlation formulation of spin Hall effect}

The relation (4) indicates that what is observed is not spin current, but its divergence. The uniform component of the response function of spin current for the applied electric field does not therefore account for the observed spin accumulation, but solving the diffusion equation is necessary. It was recently demonstrated theoretically that the spin Hall effect can be formulated by considering directly a response of spin density for the applied electric field, namely, the correlation function of velocity operator $v_{i}$ and spin $\sigma_{j}[9]$ :

$$
C_{J S}^{i j}(\boldsymbol{q}) \equiv \sum_{\boldsymbol{k}} \operatorname{Tr}\left(v_{i} G_{\boldsymbol{k}+\boldsymbol{q}}^{\mathrm{r}} \sigma_{j} G_{\boldsymbol{k}}^{\mathrm{a}}\right),
$$

where $G_{\boldsymbol{k}}^{\mathrm{r}}\left(G_{\boldsymbol{k}}^{\mathrm{a}}\right)$ is the retarded (advanced) electron Green function for wave vector $\boldsymbol{k}$ at zero angular frequency and $\boldsymbol{q}$ is an external wave vector. The analysis was carried out for the spin-orbit interaction arising from random impurities

$$
H_{\text {so }}=\lambda \int \mathrm{d}^{3} r c^{\dagger}\left[\left(\nabla V_{i}(\boldsymbol{r}) \times \boldsymbol{p}\right) \cdot \boldsymbol{\sigma}\right] c,
$$

where $c$ and $c^{\dagger}$ are electron field operators, $\boldsymbol{p}$ is electron momentum, $\lambda$ is the strength of the spin-orbit interaction, and $V_{i}(\boldsymbol{r})$ is a point-like impurity potential, $V_{i}(\boldsymbol{r})=V_{i} \delta\left(\boldsymbol{r}-\boldsymbol{R}_{i}\right)$, where $V_{i}$ is the strength. $\boldsymbol{R}_{i}$ is random impurity position, of which we take the average value. Using gradient expansion, the leading order contribution of the response function $C_{J S}^{i j}(\boldsymbol{q})$ was shown to be linear in $\boldsymbol{q}$ :

$$
C_{J S}^{i j}(\boldsymbol{q})=\mathrm{i} \lambda_{\mathrm{sh}} \epsilon_{i j k} q_{k},
$$

where $\lambda_{\mathrm{sh}}$ is a constant proportional to $\lambda$. The spin Hall effect is thus described as a spin density generated by an electric field as

$$
\boldsymbol{s}=\lambda_{\mathrm{sh}}(\nabla \times \boldsymbol{E}) .
$$

This formula indicates that the spin accumulation is formed at edges where the electric field and current are spatially inhomogeneous, namely, boundaries are essential in the spin Hall effect [10] (Fig. 1). The spin accumulation is consistent with conventional spin current expression, Eq. (1). In fact, in the present model, $\theta_{\mathrm{sh}}=\lambda_{\mathrm{sh}} / \tau$, where $\tau$ is elastic lifetime of electron. Thus the two expressions (1) and (8) satisfy the continuity Eq. (4) with $\tau_{\mathrm{r}}=\tau$. The relaxation time here coincides with $\tau$ because spin relaxation is not taken into account here.

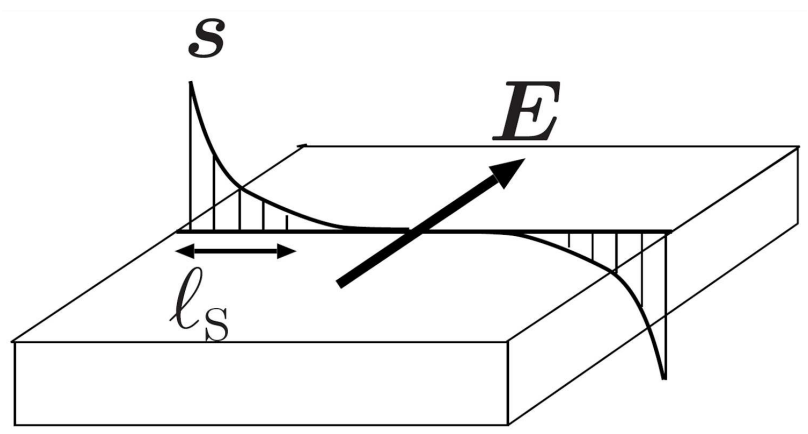

Fig. 1. Schematic figure describing the spin accumulation given by Eq. (9). The spin accumulation is formed at edges perpendicular to the applied electric field $\boldsymbol{E}$, and decays in the length scale of $\ell_{\mathrm{S}}$, the spin diffusion length. A single Eq. (9) therefore describes the conventional argument of spin Hall effect using spin current conductivity and diffusion equation.

Experimental diffusive situation is described by including electron diffusion ladder, resulting in

$$
\boldsymbol{s}=\lambda_{\mathrm{sh}} \int \mathrm{d}^{3} r^{\prime}\left[\nabla D_{\mathrm{s}}\left(\boldsymbol{r}-\boldsymbol{r}^{\prime}\right)\right] \times \boldsymbol{E}\left(\boldsymbol{r}^{\prime}\right) .
$$

Here $D_{\mathrm{s}}(\boldsymbol{r})$ is the spin diffusion propagator, the Fourier transform of

$$
D_{\mathrm{s}}(q) \equiv \frac{1}{D q^{2} \tau+\frac{4}{3} \gamma},
$$

where $\gamma \equiv \tau / \tau_{\text {so }}$ represents the strength of spin relaxation, $\tau_{\text {so }}$ being the spin lifetime due to spin-orbit interaction. $D_{\mathrm{s}}(\boldsymbol{r})$ decays exponentially in the length scale of spin diffusion length (spin relaxation length), $\ell_{\mathrm{s}}=\ell /(2 \sqrt{\gamma}), \ell$ being the electron elastic mean free path. The description provides direct relation between the physical observable and the applied field without ambiguity and is consistent with previous description using the diffusion equation. In the above formulation, the efficiency of "spin current propagation" is equivalent to ferromagnetic susceptibility. Spintronics provides therefore an electric means to measure susceptibility with spatial resolution.

In this scheme, the inverse spin Hall effect needs to be argued including the mechanism for "spin current generation". Spin pumping effect [11], one of the most widely used mechanisms for spin current generation, was recently reformulated in terms of the effective gauge field that induces spin accumulation at the ferromagnetnormal metal interface [12]. The inverse spin Hall effect for the "spin current injection" by use of spin pumping effect is therefore described by the same response function as the one for spin Hall effect, namely, $C_{J S}[9]$. 


\section{Rashba spin-orbit interaction}

In this section, we focus on the Rashba interaction and explore in detail the effects of spin-charge conversion and optical responses.

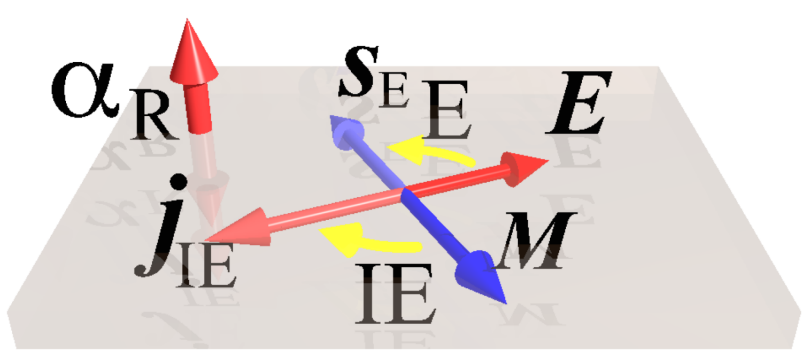

Fig. 2. Geometry of Rashba-Edelstein effect (E) and the inverse effect (IE), represented by Eqs. (12), (14).

\subsection{Spin-charge mixing and optical property}

We consider a spin-orbit interaction linear in the electron's momentum. For such an interaction to arise, spatial inversion symmetry needs to be broken. The following interaction, called the Rashba interaction, appears on surfaces and interfaces:

$$
H_{\mathrm{R}}=\mathrm{i} \boldsymbol{\alpha}_{\mathrm{R}} \cdot(\boldsymbol{\nabla} \times \boldsymbol{\sigma}) .
$$

Here a vector $\boldsymbol{\alpha}_{\mathrm{R}}$ represents an effective electric field arising from broken inversion, and is perpendicular to the interface or surface. The form of the interaction is the one derived directly from the Dirac equation as a relativistic interaction, but the magnitude can be strongly enhanced in solids having heavy elements compared to the vacuum case. The Rashba interaction mixes electron's spin and momentum in the plane perpendicular to the Rashba vector $\boldsymbol{\alpha}_{\mathrm{R}}$, resulting in spin-charge conversion effects. Evaluating the correlation function of spin and electric current, a spin density induced by an external electric field $\boldsymbol{E}$ was shown to be [13, 14] (Fig. 2):

$$
\boldsymbol{s}_{\mathrm{E}}(\omega)=\kappa_{\mathrm{E}}(\omega) \boldsymbol{\alpha}_{\mathrm{R}} \times \boldsymbol{E}(\omega) \text {. }
$$

Here $n_{\mathrm{e}}$ is electron density, and the conversion efficiency is $\kappa_{\mathrm{E}}(\omega)=-\frac{e \hbar n_{\mathrm{e}}}{m \alpha_{\mathrm{R}}^{2}} \operatorname{Im} \frac{C(\omega)}{\omega}$, where

$$
\begin{aligned}
& C(\omega) \equiv-\frac{4{\tilde{\alpha_{\mathrm{R}}}}^{2}}{n_{\mathrm{e}}} \epsilon_{\mathrm{F}} \\
& \quad \times \int \frac{\mathrm{d}^{3} k}{(2 \pi)^{3}} \sum_{\sigma= \pm} \frac{\sigma f_{\boldsymbol{k} \sigma}\left|\boldsymbol{k} \times \boldsymbol{\alpha}_{\mathrm{R}}\right|}{(\hbar \omega+\mathrm{i} \eta)^{2}-4\left(\boldsymbol{k} \times \boldsymbol{\alpha}_{\mathrm{R}}\right)^{2}},
\end{aligned}
$$

$\sigma= \pm$ denotes spin, $\tilde{\alpha_{\mathrm{R}}} \equiv \alpha_{\mathrm{R}} \equiv \frac{m \alpha}{\hbar^{2} k_{\mathrm{F}}}, f_{\boldsymbol{k} \sigma} \equiv$ $\left[\mathrm{e}^{\epsilon \boldsymbol{k} \sigma /\left(k_{\mathrm{B}} T\right)}+1\right]^{-1}$ is the Fermi distribution function, $\epsilon \boldsymbol{k} \sigma$ is the energy of electron, $\epsilon \mathrm{F}$ is the Fermi energy and $\eta$ is a damping parameter. The inverse effect is written as

$$
\boldsymbol{j}_{\mathrm{IE}}(\omega)=\mathrm{i} \omega \hbar \gamma \kappa_{\mathrm{E}}(\omega) \boldsymbol{\alpha}_{\mathrm{R}} \times \boldsymbol{B}(\omega),
$$

with the same parameter $\kappa_{\mathrm{E}}$. The effects of Eqs. (12) and (14) are called the Rashba-Edelstein effect and the inverse Rashba-Edelstein effect, respectively.
The inverse Rashba-Edelstein effect is useful for electrically detecting spin accumulation generated by spin current injection [5]. The induced spin of Eq. (12) leads to a magnetization of $\boldsymbol{M}_{\mathrm{E}} \equiv-\hbar \gamma \boldsymbol{s}_{\mathrm{E}}$, which gives rise to a magnetization current $\boldsymbol{j}_{\mathrm{E}} \equiv \nabla \times \boldsymbol{M}_{\mathrm{E}}=-\hbar \gamma \kappa_{\mathrm{E}} \nabla \times\left(\boldsymbol{\alpha}_{\mathrm{R}} \times\right.$ $\boldsymbol{E})$. This current and the inverse Rashba-Edelstein current (14), written by use of the Faraday law as $j_{\mathrm{IE}}=$ $\hbar \gamma \kappa_{\mathrm{E}} \boldsymbol{\alpha}_{\mathrm{R}} \times(\nabla \times \boldsymbol{E})$. The total current induced by the applied electric field is therefore (as function of wave vector $\boldsymbol{q}$ ):

$$
\left(j_{\mathrm{IE}}+j_{\mathrm{E}}\right)_{i}=\mathrm{i} \hbar \gamma \kappa_{\mathrm{E}}\left[q_{i} \alpha_{\mathrm{R} j}-q_{j} \alpha_{\mathrm{R} i}\right] E_{j} .
$$

This anti-symmetric conductivity linear in $\boldsymbol{q}$ indicates a rotation of the polarization of the incident electric field (directional circular dichroism). This effect acts only for circularly-polarized light and is an example of so-called natural optical activity, which is allowed when inversion symmetry is broken.

\subsection{Optical metamaterial}

Another interesting feature of the Rashba interaction is a response as a metamaterial. The combination of direct and inverse Rashba-Edelstein effects results in a current $\boldsymbol{j}_{\mathrm{IE} \cdot \mathrm{E}} \equiv \mathrm{i} \omega \hbar \gamma \kappa_{\mathrm{E}}\left(\boldsymbol{\alpha}_{\mathrm{R}} \times \boldsymbol{M}_{\mathrm{E}}\right)=-\mathrm{i} \omega\left(\hbar \gamma \kappa_{\mathrm{E}}\right)^{2}\left[\boldsymbol{\alpha}_{\mathrm{R}} \times\left(\boldsymbol{\alpha}_{\mathrm{R}} \times\right.\right.$ $\boldsymbol{E})$ ], which leads to reduction of the plasma frequency in the plane perpendicular to $\boldsymbol{\alpha}_{\mathrm{R}}$. As a result, the conductivity tensor in the long-wavelength $(\boldsymbol{q} \rightarrow 0)$ limit is $\left(\hat{\boldsymbol{\alpha}_{\mathrm{R}}} \equiv \boldsymbol{\alpha}_{\mathrm{R}} / \alpha_{\mathrm{R}}\right)$ :

$$
\begin{aligned}
& \sigma_{i j}(\omega)= \\
& \quad \frac{i e^{2}}{\omega+\mathrm{i} \eta} \frac{n_{\mathrm{e}}}{m}\left(\delta_{i j}(1+C(\omega))-\hat{\alpha}_{\mathrm{R}_{i}} \hat{\alpha}_{\mathrm{R}_{j}} C(\omega)\right) .
\end{aligned}
$$

Choosing $\boldsymbol{\alpha}_{\mathrm{R}}$ along the $z$-axis, the dielectric tensor

$$
\varepsilon_{i j}(\omega)=\delta_{i j}+\frac{1}{\varepsilon_{0}} \frac{\mathrm{i}}{\omega} \sigma_{i j}(\omega)
$$

reads

$$
\begin{aligned}
& \varepsilon_{z z}=1-\frac{\omega_{p}^{2}}{\omega(\omega+\mathrm{i} \eta)} \equiv \varepsilon_{z}, \\
& \varepsilon_{x x}=\varepsilon_{y y}=1-\frac{\omega_{p}^{2}}{\omega(\omega+\mathrm{i} \eta)}(1+C(\omega)) \equiv \varepsilon_{\perp},
\end{aligned}
$$

where $\omega_{p}=\sqrt{e^{2} n_{\mathrm{e}} / \varepsilon_{0} m}$ is the bare plasma frequency. The reduced plasma frequency in the $x y$ plane is $\omega_{\mathrm{R}} \equiv$ $\omega_{p} \sqrt{1+\operatorname{Re} C\left(\omega_{\mathrm{R}}\right)}$ ( $\operatorname{Re} C$ is negative in the frequency range we are interested).

The frequency range between the bare plasma frequency $\omega_{p}$ and the reduced plasma frequency $\omega_{\mathrm{R}}$ is of particular interest, since the system is insulating in the direction of the Rashba field but metallic in the perpendicular direction. Materials with such anisotropy are called hyperbolic because of their hyperbolic dispersion [15]. In fact, choosing the wavevector $\boldsymbol{q}$ in the $x z$ plane, the dispersion relation is $c^{2} q^{2}-\omega^{2} \varepsilon_{\perp}=0$ for $E_{y}$ and

$$
\frac{q_{z}^{2}}{\varepsilon_{\perp}}+\frac{q_{x}^{2}}{\varepsilon_{z}}=\frac{\omega^{2}}{c^{2}}
$$

for $E_{x}$ and $E_{z}$. The hyperbolic dispersion leads to the following peculiar optical properties. When the interface 

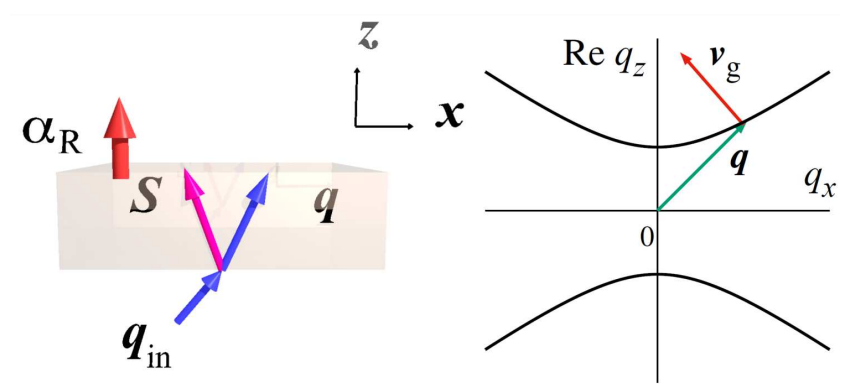

Fig. 3. (left) Schematic illustration of the geometries of electromagnetic wave incident on the Rashba system. The incident wave vector, the wave vector in the Rashba medium and the Poynting vector are denoted by $\boldsymbol{q}_{\text {in }}$, $\boldsymbol{q}$ and $\boldsymbol{s}$, respectively. (right) Dispersion curves for a fixed angular frequency and the relation between the wave vector $\boldsymbol{q}$ and the group velocity $\boldsymbol{v}_{\mathrm{g}}$ in the case of hyperbolic dispersion. They have $x$-components in the opposite directions, meaning negative refraction.

is parallel to the metallic plane, the incident light tends to be refracted in the negative direction, since the metallic nature in the $x y$-plane reflects the light. This behavior is observed in the dispersion curve (Fig. 3,right), which reveals that the $x$-component of the group velocity $\boldsymbol{v}_{\mathrm{g}}$ is negative. The Poynting vector $s$ also has a negative $x$-component.

For a bulk Rashba material of BiTeI, the maximum plasma frequency known so far is $\omega_{p}=2.5 \times 10^{14} \mathrm{~Hz}$ (corresponding to a wavelength of $7.5 \mu \mathrm{m}$ ) for $n_{\mathrm{e}}=8 \times$ $10^{25} \mathrm{~m}^{-3}$ and $\epsilon_{\mathrm{F}}=0.2 \mathrm{eV}[16]$. Using $\alpha_{\mathrm{R}}=3.85 \mathrm{eV} \AA$ And $k_{\mathrm{F}} \sim 0.1 \AA^{-1}$, we have $\tilde{\alpha_{\mathrm{R}}} \sim 1$, and thus, $C(\omega) \sim-0.4$ for frequencies $\omega \lesssim \omega_{p}$, resulting in $\omega_{\mathrm{R}} / \omega_{p}=0.77$ $\left(\omega_{\mathrm{R}}=1.9 \times 10^{14} \mathrm{~Hz}\right.$, corresponding to the wavelength of $9.8 \mu \mathrm{m}$ ). Thus, hyperbolic behavior arises in the infrared regime.

\section{Magnetic Rashba conductor}

The Rashba interaction breaking spatial inversion symmetry along the Rashba vector leads to various interesting spin transport and optical responses as we have seen. When the time reversal symmetry is broken in addition, other anomalous properties are expected. Here we discuss such a optical property considering a magnetic Rashba conductor with magnetization $\boldsymbol{M}$ (or in a magnetic field), which breaks the time-reversal symmetry. For the system lacking both spatial inversion and time-reversal symmetries, asymmetric propagation of light called the directional dichroism is allowed. We demonstrate directional dichroism based on two different approaches.

\subsection{Linear response study of conductivity}

Evaluating the conductivity at finite angular frequency $\omega$ by a linear response theory, it was shown that the magnetization and the Rashba interaction induces a component $[13,14]$ :

$$
\begin{gathered}
\sigma_{i j}^{M}=\frac{e^{2} n_{\mathrm{e}} J_{s d}}{m \epsilon_{\mathrm{F}} k_{\mathrm{F}} \omega}\left[E_{1}(\omega) \hat{\left(\boldsymbol{\alpha}_{\mathrm{R}}\right.} \times \boldsymbol{M}\right) \cdot \boldsymbol{q}\left[\delta_{i j}-\hat{\alpha}_{\mathrm{R} i} \hat{\alpha}_{\mathrm{R}_{j}}\right] \\
\left.-\frac{E_{3}(\omega)}{2}\left[M_{i}^{\perp}\left(\hat{\boldsymbol{\alpha}_{\mathrm{R}}} \times \boldsymbol{q}\right)_{j}+\left(\hat{\boldsymbol{\alpha}_{\mathrm{R}}} \times \boldsymbol{q}\right)_{i} M_{j}^{\perp}\right]\right],
\end{gathered}
$$

where $\boldsymbol{M}^{\perp}=\boldsymbol{M}-\left(\boldsymbol{M} \cdot \hat{\boldsymbol{\alpha}_{\mathrm{R}}}\right) \hat{\boldsymbol{\alpha}_{\mathrm{R}}}$ and $E_{1}$ and $E_{3}$ are coefficient defined in Ref. [13]. Terms having diagonal components linear in the wave vector $\boldsymbol{q}$ induce directional dichroism when $\boldsymbol{\alpha}_{\mathrm{R}} \times \boldsymbol{M}$ is finite (in Eq. (18) we have kept only the contribution to the directional dichroism). Considering the case where the Rashba field is in the $z$ direction with the magnetization along the $y$-axis (Fig. 4), the dispersion relation including Eq. (18) for the incident light in the $x z$ plane having the electric field in the $y$-direction reads

$$
q \simeq \frac{\omega}{c} \sqrt{\varepsilon_{\perp}}+\frac{1}{2} \frac{\omega_{\mathrm{p}}^{2}}{c^{2}} \frac{J_{s d} M}{k_{\mathrm{F}} \varepsilon_{\mathrm{F}} q} E_{13}\left(\boldsymbol{q} \cdot \boldsymbol{u}_{\mathrm{R}}\right),
$$

where $E_{13} \equiv E_{1}+E_{3}$ and

$$
\boldsymbol{u}_{\mathrm{R}} \equiv\left(\boldsymbol{\alpha}_{\mathrm{R}} \times \boldsymbol{M}\right)
$$

is a moment that governs the dichroism.

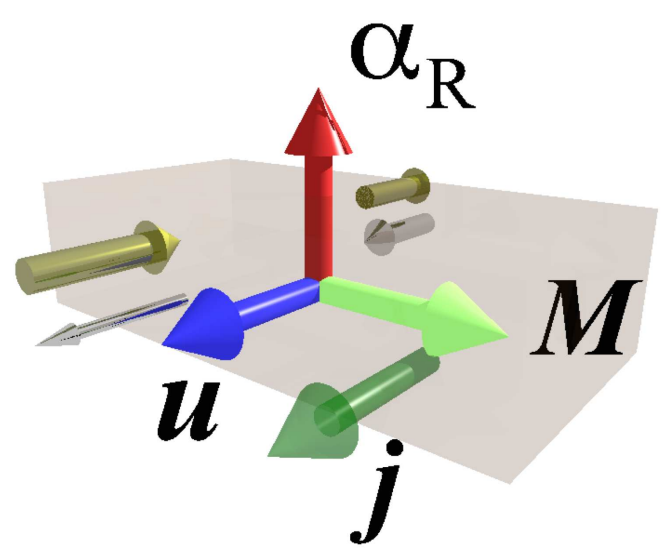

Fig. 4. Geometry of directional dichroism induced in a conductor having the Rashba interaction and magnetization or magnetic field. The directional dichroism is induced when a "scalar chirality" of the three vectors $\boldsymbol{q} \cdot\left(\boldsymbol{\alpha}_{\mathrm{R}} \times \boldsymbol{M}\right)$ is finite. The quantity $\boldsymbol{u}_{\mathrm{R}} \equiv\left(\boldsymbol{\alpha}_{\mathrm{R}} \times \boldsymbol{M}\right)$ is an effective gauge field for light, or a toroidal moment. It turns out that the same vector $\boldsymbol{u}_{\mathrm{R}}$ acts as a gauge field for electron spin (spin gauge field) (Eq. (29)), inducing a spin-polarized current $\boldsymbol{j}$ when dynamic.

\subsection{Effective Hamiltonian of electromagnetic fields}

The optical response can be studied based on an effective Hamiltonian for electromagnetic field calculated by integrating out conduction electron in the imaginarytime path integral formalism. It was demonstrated in Ref. [17] that the effective Hamiltonian including the magnetization to the linear order is

$$
\begin{gathered}
H_{E M}^{\mathrm{R}}=g \int \mathrm{d}^{3} r \boldsymbol{u}_{\mathrm{R}} \cdot(\boldsymbol{E} \times \boldsymbol{B}) \\
+\lambda_{\mathrm{R}} \int \mathrm{d}^{3} r \sum_{\mu \nu} Q_{\mu \nu} E_{\mu} B_{\nu},
\end{gathered}
$$


where $Q_{\mu \nu} \equiv M_{\mu}^{\perp} \alpha_{\mathrm{R}, \nu}$ is the effective quadrupole moment, $g$ and $\lambda_{\mathrm{R}}$ are constants and $\boldsymbol{E}$ and $\boldsymbol{B}$ are the electric and magnetic fields, respectively.

The first term of $H_{E M}^{\mathrm{R}}$ has a clear physical meaning of the Doppler shift. In fact, the vector $\frac{1}{\mu} \boldsymbol{E} \times \boldsymbol{B} \equiv \boldsymbol{q}$ (where $\mu$ is the magnetic permeability of solids) is the Poynting vector representing the momentum of the electromagnetic wave, and the vector interaction of Eq. (21) is of the form representing the Doppler shift $\boldsymbol{u}_{\mathrm{R}} \cdot \boldsymbol{q}$. The vector $\boldsymbol{u}_{\mathrm{R}}$ therefore acts as an effective gauge field (vector potential) for electromagnetic waves, that induces an intrinsic velocity of the medium. In multiferroic (insulating) systems, the vector product of electric polarization $\boldsymbol{p}$ and magnetization, $\boldsymbol{p} \times \boldsymbol{M}$, has been discussed to induce directional dichroism and the Doppler shift of light based on a symmetry argument [18]. References [13, 17] provided microscopic justification of this fact in the conducting case with $\boldsymbol{p}$ replaced by $\boldsymbol{E}$.

The Doppler shift picture is clearly seen from the equation of motion including the vector interaction (the first term of Eq. (21)). In fact, the total electric and magnetic fields defined from the equation of motion are [17]:

$$
\begin{gathered}
\boldsymbol{E}_{\mathrm{tot}}=\boldsymbol{E}+\frac{1}{\epsilon_{0}}\left(\boldsymbol{u}_{\mathrm{R}} \times \boldsymbol{B}\right), \\
\boldsymbol{B}_{\mathrm{tot}}=\boldsymbol{B}+\mu_{0}\left(\boldsymbol{u}_{\mathrm{R}} \times \boldsymbol{E}\right) .
\end{gathered}
$$

The cross-correlation effect in magnetic Rashba conductor, therefore, is considered as a result of a Lorentz transformation to a moving frame with velocity $\boldsymbol{u}_{\mathrm{R}}$. We have, using $\frac{\partial \boldsymbol{B}}{\partial t}=-\boldsymbol{\nabla} \times \boldsymbol{E}$ :

$$
\frac{\partial \boldsymbol{E}_{\mathrm{tot}}}{\partial t}=\frac{\partial \boldsymbol{E}}{\partial t}+\frac{1}{\epsilon_{0}}[(\boldsymbol{u} \cdot \boldsymbol{\nabla}) \boldsymbol{E}-\boldsymbol{\nabla}(\boldsymbol{u} \cdot \boldsymbol{E})],
$$

which for plane waves with the wave vector $\boldsymbol{k} \perp \boldsymbol{E}$ is represented as a "covariant" derivative,

$$
D_{t} \equiv \frac{\partial}{\partial t}+\frac{1}{\epsilon_{0}}(\boldsymbol{u} \cdot \boldsymbol{\nabla}),
$$

which is expected for a flowing medium [19]. Therefore, the electromagnetic cross-correlation effect shown in Eq. (28) represents the Doppler shift because of a medium flow with velocity $\boldsymbol{u}_{\mathrm{R}}$.

\subsection{Weyl-type spin-orbit interaction}

We briefly mention the case of another spin-orbit interaction

$$
\mathcal{H}_{\mathrm{W}}=-\lambda_{\mathrm{W}}(\boldsymbol{p} \cdot \boldsymbol{\sigma}),
$$

where $\lambda_{\mathrm{W}}$ is a coupling constant. We do not consider magnetization here. Hamiltonian (25) breaks inversion symmetry in any spatial directions but keeps the timereversal invariance. Such a system is called chiral [20]. The effective Hamiltonian for electromagnetic field in this case is obtained as [21]:

$$
H_{E B}^{\mathrm{W}}=g_{\mathrm{W}} \int \mathrm{d}^{3} r(\boldsymbol{B} \cdot \dot{\boldsymbol{E}}-\boldsymbol{E} \cdot \dot{\boldsymbol{B}}),
$$

where $g_{\mathrm{W}}$ is a constant. The quantity on the right-hand side is a measure of chirality of electromagnetic field. Using the Maxwell equation in vacuum, it reads

$$
H_{E B}^{\mathrm{W}}=g_{\mathrm{W}} \int \mathrm{d}^{3} r\left[c^{2} \boldsymbol{B} \cdot(\nabla \times \boldsymbol{B})+\boldsymbol{E} \cdot(\nabla \times \boldsymbol{E})\right] .
$$

The total fields read

$$
\begin{aligned}
& \boldsymbol{E}_{\mathrm{tot}} \equiv \boldsymbol{E}-\frac{g_{\mathrm{W}}}{\epsilon_{0}} \dot{\boldsymbol{B}}=\boldsymbol{E}+\frac{g_{\mathrm{W}}}{\epsilon_{0}} \nabla \times \boldsymbol{E}, \\
& \boldsymbol{B}_{\mathrm{tot}} \equiv \boldsymbol{B}-\mu_{0} g_{\mathrm{W}} \dot{\boldsymbol{E}}=\boldsymbol{B}-\frac{g_{\mathrm{W}}}{\epsilon_{0}} \nabla \times \boldsymbol{B} .
\end{aligned}
$$

The chiral meaning of $H_{E B}^{\mathrm{W}}$ is clear from these expressions connecting $\boldsymbol{E}$ and $\nabla \times \boldsymbol{E}$.

\subsection{Effective gauge field for electron spin}

The Rashba interaction (11), being linear in the electron's momentum, can be approximated as a gauge interaction to the linear order. Namley, $H_{\mathrm{R}}=\boldsymbol{p} \cdot\left(\boldsymbol{\alpha}_{\mathrm{R}} \times \boldsymbol{\sigma}\right)$, where $\left(\boldsymbol{\alpha}_{\mathrm{R}} \times \boldsymbol{\sigma}\right)$ plays a role of a gauge field. Being proportional to the Pauli matrix, its effect becomes essential in the spin-polarized case of metallic ferromagnets. We consider the case of strong $s d$ exchange coupling, where the spin operator $\boldsymbol{\sigma}$ can be replaced by $\boldsymbol{n}$, a unit vector along the localized spin. We thus have an U(1) effective gauge field for electron spin [22, 23]:

$$
\boldsymbol{A}_{\mathrm{R}} \equiv-\frac{m}{e \hbar}\left(\boldsymbol{\alpha}_{\mathrm{R}} \times \boldsymbol{n}\right) .
$$

Existence of a gauge field naturally leads to an effective electric and magnetic fields

$$
\begin{aligned}
& \boldsymbol{E}_{\mathrm{R}}=-\dot{\boldsymbol{A}}_{\mathrm{R}}=\frac{m}{e \hbar}\left(\boldsymbol{\alpha}_{\mathrm{R}} \times \dot{\boldsymbol{n}}\right), \\
& \boldsymbol{B}_{\mathrm{R}}=\boldsymbol{\nabla} \times \boldsymbol{A}_{\mathrm{R}}=-\frac{m}{e \hbar} \boldsymbol{\nabla} \times\left(\boldsymbol{\alpha}_{\mathrm{R}} \times \boldsymbol{n}\right) .
\end{aligned}
$$

It was shown that electron spin relaxation induces a perpendicular component of effective electric field [24]:

$$
\boldsymbol{E}_{\mathrm{R}}^{\prime}=\frac{m}{e \hbar} \beta_{\mathrm{R}}\left[\boldsymbol{\alpha}_{\mathrm{R}} \times(\boldsymbol{n} \times \dot{\boldsymbol{n}})\right],
$$

where $\beta_{\mathrm{R}}$ is a coefficient representing the strength of spin relaxation. For the case of strong Rashba interaction of $\alpha_{\mathrm{R}}=3 \mathrm{eV} \AA$, as realized in $\mathrm{Bi} / \mathrm{Ag}$, the magnitude of the electric field is $\left|E_{\mathrm{R}}\right|=\frac{m}{e \hbar} \alpha_{\mathrm{R}} \omega=26 \mathrm{kV} / \mathrm{m}$ if the angular frequency $\omega$ of magnetization dynamics is $10 \mathrm{GHz}$. The magnitude of relaxation contribution is $\left|E_{\mathrm{R}}^{\prime}\right| \sim 260 \mathrm{~V} / \mathrm{m}$ if $\beta_{\mathrm{R}}=0.01$. The effective magnetic field in the case of spatial length scale of $10 \mathrm{~nm}$ is $B_{\mathrm{R}} \sim 260 \mathrm{~T}$, and we expect large spin Hall effect by applying an electric field along the Rashba vector.

The Rashba-induced electric fields, $\boldsymbol{E}_{\mathrm{R}}$ and $\boldsymbol{E}_{\mathrm{R}}^{\prime}$, are important from the viewpoint of spin-charge conversion. In fact, results (30),(31) indicate that a voltage is generated by a dynamics magnetization if the Rashba interaction is present. Importantly, this effect emerges even from a spatially uniform magnetization precession, in sharp contrast to the conventional adiabatic effective electric field (spin motive force), arising from the combination of spatial and temporal variations. In the case of a thin film with the Rashba interaction perpendicular to the plane and with a precessing magnetization, the component $\boldsymbol{E}_{\mathrm{R}} \propto \dot{\boldsymbol{n}}$ has no DC component, while the relaxation contribution $\boldsymbol{E}_{\mathrm{R}}^{\prime}$ has a DC component perpendicular to $\overline{\boldsymbol{n} \times \dot{\boldsymbol{n}}} \| \overline{\boldsymbol{n}}$, where $\overline{\boldsymbol{n} \times \dot{\boldsymbol{n}}}$ and $\overline{\boldsymbol{n}}$ denote 
time-averages. The geometry of this current pumping effect, $\boldsymbol{j} \propto \boldsymbol{E}_{\mathrm{R}}^{\prime} \propto \boldsymbol{\alpha}_{\mathrm{R}} \times \overline{\boldsymbol{n}}$ (Fig. 5), is therefore the same as the one expected in the case of inverse Edelstein effect, conventionally discussed in terms of spin current.

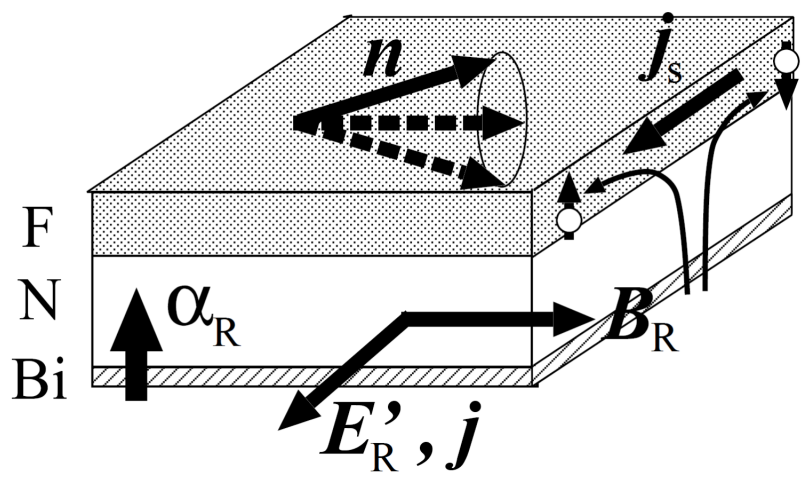

Fig. 5. Schematic figure depicting Rashba-induced spin electric and magnetic fields $\boldsymbol{E}_{\mathrm{R}}^{\prime}$ and $\boldsymbol{B}_{\mathrm{R}}$ generated by magnetization precession in a junction of a ferromagnet $(\mathrm{F})$, a nonmagnetic spacer $(\mathrm{N})$ and a heavy atom layer $(\mathrm{Bi})$, where the Rashba interaction is induced. The component $\boldsymbol{E}_{\mathrm{R}}^{\prime}$ induces a current $\boldsymbol{j}$ for precessing magnetization vector $\boldsymbol{n}$ in the direction perpendicular to both $\boldsymbol{n} \times \dot{\boldsymbol{n}}$ and Rashba field $\boldsymbol{\alpha}_{\mathrm{R}}$, and has DC component as the average of $\boldsymbol{n} \times \dot{\boldsymbol{n}}$ is finite. $\boldsymbol{E}_{\mathrm{R}} \propto \dot{\boldsymbol{n}}$ has only AC. The magnetic field component $\boldsymbol{B}_{\mathrm{R}}$ lies in-plane and is expected to induce "giant" spin Hall effect $\left(\boldsymbol{j}_{\mathrm{s}}\right)$ when an electric field is applied perpendicular to the plane.

In the present form, Eqs. (30), (31), the Rashbainduced electric field is a local quantity; a voltage is generated by a direct contact between the Rashba interaction and magnetization. It becomes long-ranged if electron diffusion is taken into account as pointed out recently [25], and the inverse Edelstein effect with an Ag spacer layer, would be explained by the long-ranged Rashba-induced voltage.

To detect the magnetic component, $\boldsymbol{B}_{\mathrm{R}}$, which can be of the order of $100 \mathrm{~T}$, is essential for the confirmation of gauge field scenario. In the setup of Fig. $5, \boldsymbol{B}_{\mathrm{R}}$ is along $\overline{\boldsymbol{n}}$. The field can therefore be detected by measuring "giant" in-plane spin Hall effect when a current is injected perpendicular to the plane.

General relation between spin and charge dynamics in the presence of spin-orbit interaction in two-dimensional electron gas was discussed in Ref. [26]. Electric current generation by spin dynamics with Rashba spin-orbit interaction was theoretically discussed in the case of a $\operatorname{dot}[27]$.

\section{Summary}

A theoretical overview of spin-charge conversion effects due to spin-orbit interactions has been presented. We first argued that the spin Hall effect can be described in terms of a single correlation function between physical spin density and electric current, avoiding ambiguity of spin current and use of classical diffusion equation in addition to linear response theory for spin current. Spin-charge conversion effects indicates intriguing cross correlation effects in electromagnetism, and these effects are described universally by use of effective gauge fields. What is striking is the fact that the effective gauge field for electron spin, $\boldsymbol{A}_{\mathrm{R}}$ of Eq. (29), is identical (up to numerical coefficients) to the one for photons, $\boldsymbol{u}_{\mathrm{R}}$ of Eq. (20). Although allowed by symmetry, this is a nontrivial fact indicating generality and usefulness of the concept of effective gauge field.

\section{Acknowledgments}

This work was supported by a Grant-in-Aid for Exploratory Research (No. 16K13853) and a Grant-in-Aid for Scientific Research (B) (No. 17H02929) from the Japan Society for the Promotion of Science and a Grantin-Aid for Scientific Research on Innovative Areas (No. 26103006) from The Ministry of Education, Culture, Sports, Science and Technology (MEXT), Japan. G.T. is grateful for the Graduate School Materials Science in Mainz (MAINZ) for financial support (DFG GSC 266).

\section{References}

[1] E. Saitoh, M. Ueda, H. Miyajima, G. Tatara, Appl. Phys. Lett. 88, 182509 (2006).

[2] E. Rashba, Sov. Phys.-Solid State 2, 1109 (1960).

[3] M. Dyakonov, V.I. Perel, Sov. Phys.-JETP Lett. 13, 467 (1971).

[4] V. Edelstein, Solid State Commun. 73, 233 (1990).

[5] J.C.R. Sanchez, L. Vila, G. Desfonds, S. Gambarelli, J.P. Attane, J.M. De Teresa, C. Magen, A. Fert, Nat. Commun. 4, 2944 (2013).

[6] K. Shen, G. Vignale, R. Raimondi, Phys. Rev. Lett. 112, 096601 (2014).

[7] Y. Kato, R.C. Myers, A.C. Gossard, D.D. Awschalom, Science 306, 1910 (2004).

[8] J. Shibata, H. Kohno, Phys. Rev. B 84, 184408 (2011).

[9] G. Tatara, Phys. Rev. B 98, 174422 (2018).

[10] V.M. Galitski, A.A. Burkov, S.D. Sarma, Phys. Rev. $B$ 74, 115331 (2006).

[11] Y. Tserkovnyak, A. Brataas, G.E.W. Bauer, Phys. Rev. Lett. 88, 117601 (2002).

[12] G. Tatara, S. Mizukami, Phys. Rev. B 96, 064423 (2017).

[13] J. Shibata, A. Takeuchi, H. Kohno, G. Tatara, J. Phys. Soc. Jpn. 85, 033701 (2016).

[14] J. Shibata, A. Takeuchi, H. Kohno, G. Tatara, J. Appl. Phys. 123, 063902 (2018).

[15] E.E. Narimanov, A.V. Kildishev, Nat. Photon. 9, 214 (2015).

[16] L. Demkó, G.A.H. Schober, V. Kocsis, M.S. Bahramy, H. Murakawa, J.S. Lee, I. Kézsmárki, R. Arita, N. Nagaosa, Y. Tokura, Phys. Rev. Lett. 109, 167401 (2012). 
[17] H. Kawaguchi, G. Tatara, Phys. Rev. B 94, 235148 (2016).

[18] K. Sawada, N. Nagaosa, Phys. Rev. Lett. 95, 237402 (2005).

[19] U. Leonhardt, P. Piwnicki, Phys. Rev. A 60, 4301 (1999).

[20] D.M. Lipkin, J. Math. Phys. 5, 696 (1964).

[21] H. Kawaguchi, G. Tatara, J. Phys. Soc. Jpn. 87, 064002 (2018).

[22] K.W. Kim, J.H. Moon, K.J. Lee, H.W. Lee, Phys. Rev. Lett. 108, 217202 (2012).
[23] N. Nakabayashi, G. Tatara, New J. Phys. 16, 015016 (2014); G. Tatara, Physica E Low-Dimens. Syst. Nanostruct. 106, 208 (2019).

[24] G. Tatara, N. Nakabayashi, K.J. Lee, Phys. Rev. B 87, 054403 (2013).

[25] J. Fujimoto, G. Tatara, Phys. Rev. B 99, 054407 (2019).

[26] I.V. Tokatly, E.Y. Sherman, Phys. Rev. B 82, 161305 (2010).

[27] L.S. Levitov, E.I. Rashba, Phys. Rev. B 67, 115324 (2003). 